\title{
Considerações sobre a avaliação do bem-estar fetal
}

Considerations about the evaluation of fetal well-being

\begin{tabular}{|c|c|c|}
\hline $\begin{array}{l}\text { Artigo } \\
\text { Original }\end{array}$ & Júlio Aragão 1 & \\
\hline \multirow{6}{*}{$\begin{array}{c}\text { Original } \\
\text { Paper }\end{array}$} & Palavras-chaves: & Resumo \\
\hline & Gravidez de Alto & A avaliação do bem-estar fetal é assunto de interesse na prática obstétrica, \\
\hline & Risco & $\begin{array}{l}\text { sendo seu impacto, na melhoria da assistência, inquestionável. Partindo desta } \\
\text { premissa, o presente trabalho apresenta considerações sobre a avaliação do }\end{array}$ \\
\hline & Monitorização & bem-estar fetal de forma a permitir ao profissional assistente uma melhor \\
\hline & Fetal & $\begin{array}{l}\text { utilizaçã3seus recursos diagnósticos. As indicações clínicas e melhores } \\
\text { oportunidades de realizacão, diferentes métodos passíveis de utilizacão }\end{array}$ \\
\hline & Sofrimento Fetal & $\begin{array}{l}\text { na avaliação, e diferentes prognósticos fetais nas situações encontradiças } \\
\text { no dia-a-dia são também abordadas. Os diferentes métodos analisados são } \\
\text { apresentados de forma a permitir sua melhor utilização na prática clínica } \\
\text { diária com impacto positivo nos indicadores de saúde perinatal. }\end{array}$ \\
\hline
\end{tabular}

Abstract

The evaluation of fetal well-being is a subject of interest in obstetrician practice, being unquestionable its impact in the assistance improvement. Starting from this premise, the present work presents considerations about the evaluation of fetal well-being in a way of allowing the assistant professional a better use of his/her diagnostic resources. The clinical indications and better opportunities of realization, different methods susceptible of using in the evaluation, and different fetal prognostics in daily situations are also approached. The different methods analyzed are presented in a way to allow their better use in the daily clinical practice with a positive impact in the perinatal health indicators.
Key words:

High-Risk Pregnancy

Fetal Monitoring

Fetal Distress

\section{Introdução}

A história da avaliação do bem estar fetal se iniciou no ano de 1821, quando Mayor anunciou a ausculta de batimentos cardíacos fetais intra-uterinos (RIBEMONTDESSAIGNES \& LEPAGE, 1923) que permaneceu por mais de um século como a principal (somada à movimentação fetal) forma de acesso à saúde fetal disponível aos clínicos.

Na medida em que ocorria redução da mortalidade perinatal e o cuidado intensivo neonatal progredia, a avaliação do bemestar fetal passou a ter maior importância clínica, permitindo o acompanhamento das condições fetais e a intervenção no momento apropriado.
Nos últimos cinqüenta anos, novas formas de avaliação do bem- estar fetal foram descritas, sendo divididas em formas clínicas, bioquímicas e biofísicas de avaliação. O presente trabalho apresentará uma rápida análise dos métodos clínicos e biofísicos de avaliação, visto que a utilização de métodos bioquímicos (mormente a dosagem de estriol urinário de 24 horas) está hoje em desuso na prática clínica.

\section{Avaliação Clínica}

Embora seja a prova imediata de vida intra-uterina, a ausculta de batimentos

${ }^{1}$ Doutor - Medicina - UniFOA 
cardíacos de forma isolada em pouco contribui para inferir qualquer prognóstico de vitalidade fetal, servindo apenas como prova de vitalidade momentânea. Por outro lado, o Teste do Estímulo Sonoro Simplificado (TESS) pode ser indicativo de boa vitalidade fetal, ainda que por um período restrito. O teste é realizado com a gestante em repouso, através de ausculta de BCFs basais seguida de estímulo sonoro de aproximadamente 5 a 10 segundos (feito por meio de uma buzina de bicicleta ou campainha domiciliar tipo "cigarra") e nova ausculta após a realização do estímulo. $\mathrm{O}$ incremento de pelo menos 15 batimentos na freqüência de base que se mantenha no mínimo por 15 segundos configura boa vitalidade fetal (fig. 01). O Mobilograma ou Registro Diário de Movimentação Fetal também pode ser utilizado, sendo que será solicitado à gestante que permaneça em repouso por 60 minutos registrando em papel a movimentação fetal deste período. A presença de um número mínimo de 06 movimentos fetais caracteriza bem estar (BRASIL, 2005).

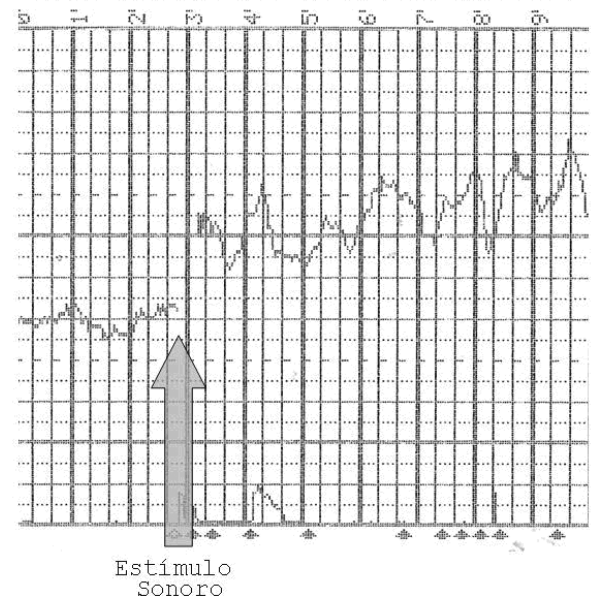

Figura 01: Aceleração dos BCFs após estímulo sonoro

\section{Avaliação biofísica}

Os avanços no campo da ultrasonografia fizeram com que a avaliação intrauterina do concepto pudesse ser realizada não somente em sua morfologia e desenvolvimento, mas também em relação a presença (ou pelo menos presunção) de hipóxia, o que propiciou a sua utilização para avaliação fetal.

Cardiotocografia

Consiste este método em um registro contínuo da atividade uterina e da variabilidade da freqüência cardíaca fetal em determinado tempo (geralmente 20 minutos) sendo método barato, de fácil execução e inócuo. A cardiotocografia avalia múltiplos parâmetros, sendo realizada tanto em repouso (cardiotocografia basal) quanto em pacientes em trabalho de parto (cardiotocografia intraparto). A utilização de sobrecarga com ocitocina (Prova de Pose) é de indicação excepcional.

O traçado cardiotocográfico é avaliado em primeira instância pela freqüência cardíaca de base, sendo o valor normal da mesma entre 120 a 160 batimentos por minuto. Valores acima ou abaixo destes configurarão taquicardia ou bradicardia fetal. Conceptos apresentando taquicardia mantida deverão ser investigados para a possibilidade de febre ou infecção materna, uso de medicações beta agonistas, ou sofrimento fetal. Em relação à bradicardia mantida, principalmente em conceptos distantes do termo da gravidez, deve-se considerar sempre a possibilidade de bloqueio de condução AV com ou sem concomitância de malformações cardíacas.

O padrão de linha de base (fig. 02) é avaliado de acordo com a amplitude de osciliações que se apresentam no concepto em repouso, ou seja, na ausência de contrações ou movimentação fetal. Segundo o Royal College of Obstetrician and Gynaecology (2001), amplitudes situadas entre 10 e 25 bpm (padrão ondulatório) são consideradas normais ou indicativas de um bom padrão de bem estar fetal. Padrões com amplitude superior a 25 bpm (padrão saltatório) são freqüentemente associadas com compressão funicular, principalmente pela presença de oligohidramnia. Padrões com amplitude inferior a $10 \mathrm{bpm}$ (padrão comprimido) geralmente indicam perído de sono no ciclo sono-vigília fetal (que podem ocorrer fisiologicamente ou por sedação fetal) ou hipóxia, sendo que amplitudes abaixo de 05 bpm estão relacionadas com hipóxia grave (padrão liso ou terminal) .
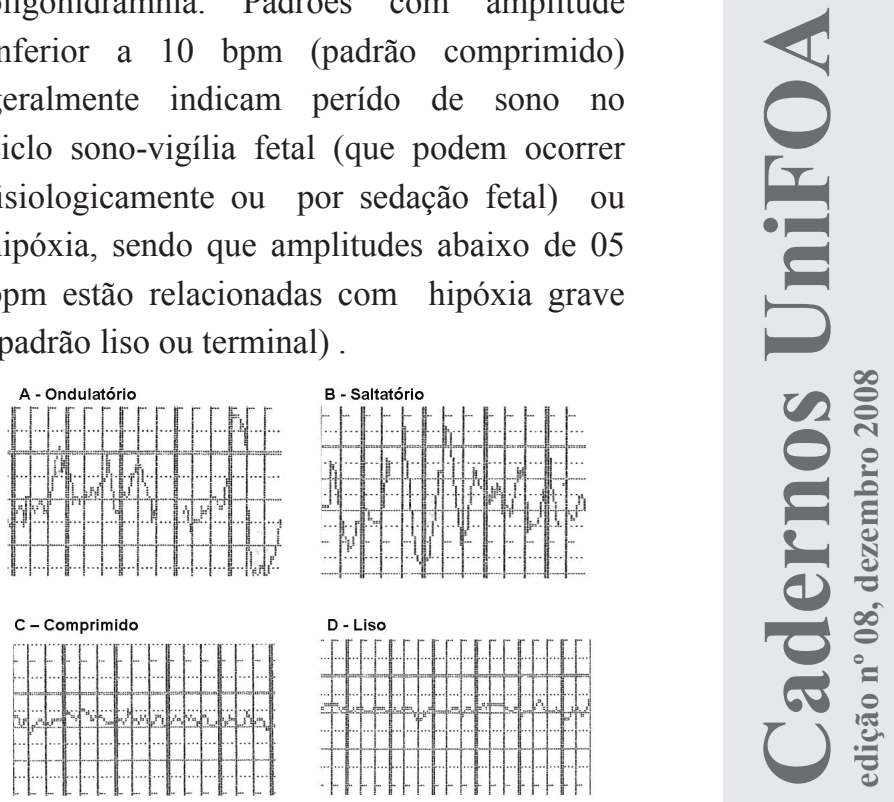

Figura 02: Padrões de Lina de Base na Cardiotocografia 
A presunção de boa vitalidade fetal poderá ser feita evidenciando episódios de acelerações transitórias da FCF em associação à movimentação fetal (complexo reatividade) em número de, no mínimo 03 em um período de 10 minutos. Em conceptos que não apresentem complexos ou que os apresentem em quantidade suficente, estará indicada a realização de estímulo vibro-acústico, que se seguirá de aceleração característica, configurando a reatividade fetal. Caso a reatividade não seja obtida na primeira tentativa, preconiza-se um novo estímulo após 05 minutos e somente então esta CTG poderá ser caracterizada como não reativa.

\subsection{Perfil Biofísico Fetal}

A combinação de alguns parâmetros da ultra-sonografia com a cardiotocografia resultaram no Perfil Biofísico Fetal (PBF), composto dos seguintes critérios com pontuação de 0 a 2: quantidade de líquido amniótico, movimentos respiratórios fetais, tônus fetal, movimentação fetal e cardiotocografia. $\mathrm{O}$ escore é compatível com boa vitalidade fetal quando atinge valor igual ou superior a 8 . Valores entre 6 e 8 indicam nova avaliação em 24 horas e valores abaixo de 6 indicam a interrupção da gestação. O PBF é um método que demanda tempo, duplicidade de equipamentos (cardiotocógrafo e ultrasom) e de profissionais. Atualmente, de uso excepcional, foi plenamente substituído pela dopplervelocimetria.

\subsection{Doppler fetal}

Embora o efeito Doppler nada tenha de novidade (descrito em 1842) seu uso na ultra-sonografia possibilitou o acesso à quantificação de parâmetros antes impossíveis, como as alterações de fluxo sanguíneo dos vasos fetais. A comparação entre os fluxos (ou a resistência ao fluxo) das artérias umbilical e cerebral média, possibilita identificar o mecanismo de centralização fetal, no qual o feto promove vasodilatação para cérebro, coração e supra-renais ao mesmo tempo em que realiza vasoconstricção nos demais órgãos (KARSDORP et al., 1994). Ao considerarmos os fluxos fisiológicos na artéria umbilical e na artéria cerebral média, fica claro que a primeira, principalmente em virtude de seu maior calibre, possui menor resistência que a segunda. Assim sendo, instalado o processo de centralização fetal, evidencia-se decréscimo de resistência ao fluxo cerebral em contraponto ao aumento evidenciado na resistência umblical, decorrente da vasoconstricção periférica e vaosodilatação das artéria do Sistema Nervoso Central. Esse parâmetro é avaliado pelo índice umbílico cerebral, que constitui relação entre os índices fluxométricos da artéria umbilical e cerebral média. Assim sendo, em situações de boa vitalidade fetal a resistência da artéria umbilical será maior que o fluxo da cerebral média, resultando sempre em índices maiores que 1. Em situações de centralização fetal instalada o fluxo sempre será menor que 1 (NOMURA et al., 2003).

Especial atenção deverá também ser dedicada a alguns aspectos relativos ao Doppler de artéria umbilical. O aumento de resistência imposto pelo processo de centralização fetal poderá criar reduções gradativas no fluxo diastólico deste vaso, podendo resultar em fluxo diastólico ausente (diástole zero) ou mesmo retrógrado (diástole reversa). A evidência dessas duas alterações indica, per se, insuficiência placentária grave, necessitando atenção intensiva por parte da equipe de assistência (FRANCISCO \& ZUGAIB, 2008).

\section{Considerações Finais}

A avaliação do bem estar fetal em muito progrediu nos últimos anos, propiciando à equipe de cuidado perinatal maiores informações sobre as condições fetais, permitindo a manutenção da gestação quando possível e indicando o a interrupção da gravidez em momento oportuno.

Ao avaliar clinicamente gestações de alto risco, as equipes multiprofissionais de cuidado perinatal deverão sempre ter em mente que o tratamento apropriado passa pela indicação correta do momento de antecipação do parto, evitando a prematuridade iatrogênica e aumentando as chances de sobrevida do recém nado. Para tal, a combinação dos diferentes métodos ora expostos, em consonância com as realidades locais de disponibilidade (e acesso) de tecnologia e cuidado neonatal serão os princípios norteadores para a redução da mortalidade perinatal. 
RIBEMONT-DESSAIGNES, A. \& LEPAGE, G. Traité d'Obstétrique. Paris, Masson et cie., 1923.

BRASIL, Ministério da Saúde. Prénatal e puerpério Atenção qualificada e humanizada - Manual Técnico, 2005

ROYAL COLLEGE OF OBSTETRICIAN AND GYNAECOLOGY . Eletronic Fetal Monitoring. The use and the interpretation of cardiotocography in intrapatum fetal surveillance. Evidence-based Guideline Number 8, 2001.

NOMURA, RMY; FRANCISCO, RPV; MIYADAHIRA, S; ZUGAIB, M. Cardiotocografia em gestações com diástole zero ou reversa nas artérias umbilicais: análise dos resultados perinatais Rev. Assoc. Med. Bras. v.49, n.1, 2003.

FRANCISCO, RPV; ZUGAIB, M. Análise crítica da dopplervelocimetria para avaliação da vitalidade fetal. Rev. Bras. Ginecol. Obstet., Rio de Janeiro, v. 30, n. 4, 2008 .

KARSDORP, V. H. M.; VAN VUGT, J. M. G.; VAN GEIJN, H. P. Clinical significance of absent or reversed end diastolic velocity waveforms in umbilical artery. Lancet v. 344, 1994.

Endereço para Correspondência:

Doutor Júlio Aragão

Curso de Medicina

julio.aragao@foa.org.br

Centro Universitário de Volta Redonda

Campus Três Poços

Av. Paulo Erlei Alves Abrantes, $n^{\circ}$ 1325,

Três Poços - Volta Redonda / RJ

CEP: 27240-560

Informações bibliográficas:

Conforme a NBR 6023:2002 da Associação Brasileira de Normas Técnicas (ABNT), este texto científico publicado em periódico eletrônico deve ser citado da seguinte forma: 